\title{
Graph Orientations and Linear Extensions.
}

\author{
Benjamin Iriarte $]^{*}$ \\ Department of Mathematics, Massachusetts Institute of Technology, Cambridge MA, USA
}

\begin{abstract}
Given an underlying undirected simple graph, we consider the set of all acyclic orientations of its edges. Each of these orientations induces a partial order on the vertices of our graph, and therefore we can count the number of linear extensions of these posets. We want to know which choice of orientation maximizes the number of linear extensions of the corresponding poset, and this problem is solved essentially for comparability graphs and odd cycles, presenting several proofs. We then provide an inequality for general graphs and discuss further techniques.

Résumé. Etant donné un graphe simple non orienté, nous considérons l'ensemble des orientations acycliques de ses arêtes. Chacune de ces orientations induit un ordre partiel sur les sommets de notre graphe, et nous pouvons donc compter le nombre d'extensions linéaires de ces ensembles ordonnés. Nous voulons savoir quel choix d'orientation maximise le nombre d'extensions linéaires de l'ensemble ordonné correspondant, et ce problème est résolu essentiellement pour les graphes de comparabilité et les cycles impairs, présentant plusieurs preuves. Nous proposons ensuite une inégalité pour les graphes généraux et nous discutons d'autres techniques.
\end{abstract}

Keywords: graph orientation, linear extension, poset, comparability graph.

\section{Introduction.}

Linear extensions of partially ordered sets have been the object of much attention and their uses and applications remain increasing. Their number is a fundamental statistic of posets, and they relate to everrecurring problems in computer science due to their role in sorting problems. Still, many fundamental questions about linear extensions are unsolved. Efficiently enumerating linear extensions of certain posets is difficult, and the general problem has been found to be $\sharp \mathrm{P}$-complete in Brightwell and Winkler (1991).

Directed acyclic graphs, and similarly, acyclic orientations of simple undirected graphs, are closely related to posets, and their problem-modeling values in several disciplines, including the biological sciences, needs no introduction. We propose the following problem:

Problem 1 Suppose that there are $n$ individuals with a known contagious disease, and suppose that we know which pairs of these individuals were in the same location at the same time. Assume that at some initial points, some of the individuals fell ill, and then they started infecting other people and so forth, spreading the disease until all $n$ of them were infected. Then, assuming no other knowledge of the situation, what is the most likely way in which the disease spread out?

*Email: biriarte@math.mit.edu.

1365-8050 (c) 2014 Discrete Mathematics and Theoretical Computer Science (DMTCS), Nancy, France 
Suppose that we have an underlying connected undirected simple graph $G=G(V, E)$ with $n$ vertices. If we first pick uniformly at random a bijection $f: V \rightarrow[n]$, and then orient the edges of $E$ so that for every $\{u, v\} \in E$ we select $(u, v)$ (read $u$ directed to $v$ ) whenever $f(u)<f(v)$, we obtain an acyclic orientation of $E$. In turn, each acyclic orientation induces a partial order on $V$ in which $u<v$ if and only if there is a directed path $\left(u, u_{1}\right),\left(u_{1}, u_{2}\right), \ldots,\left(u_{k}, v\right)$ in the orientation. In general, several choices of $f$ above will result in the same acyclic orientation. However, the most likely acyclic orientations so obtained will be the ones whose induced posets have the maximal number of linear extensions, among all posets arising from acyclic orientations of $E$. Our main problem then becomes that of deciding which acyclic orientations of $E$ attain this optimality property of maximizing the number of linear extensions of induced posets. This problem was raised by Saito (2007) for the case of trees, and a solution for the case of bipartite graphs had been obtained by Stachowiak (1988).

The main goals of this abstract will be to first solve the main problem for the cases in which $G$ is a comparability graph or an odd cycle, and then second to provide several points of view and techniques to study the general problem. In Section 2 we present a novel combinatorial proof of the main result for bipartite graphs, different to that of Stachowiak (1988) in that we explicitly construct a function that maps injectively linear extensions of non-optimal acyclic orientations to linear extensions of an optimal orientation. In Section 3 we will extend this proof to the case of odd cycles. After that, we will introduce two new techniques that will lead to different solutions for the case of comparability graphs in Section 4 These techniques will allow us to re-discover the solution for odd cycles and to state inequalities for the general enumeration problem in Section 5. The recurrences for the number of linear extensions of posets presented in Corollary 4.9 had been previously established in Edelman et al. (1989) using promotion and evacuation, but we will obtain them independently as by-products of certain network flows in Hasse diagrams. To the best of our knowledge, this is the first time that the construction of Lemma 4.8 is presented. Stachowiak (1988) had used some instances of these recurrences to solve the main problem for bipartite graphs. Finally, in Section 6, we will relate our problem to graphical arrangements and their dual graphical zonotopes, discovering that they are intimately connected.

Herein, we will identify an acyclic orientation of a simple graph with the poset that it induces. Also, when defining posets, we will try to make clear the distinction between the ground set of the poset and its order relations.

\section{The case of bipartite graphs.}

The goal of this section is to present a combinatorial proof that the number of linear extensions of a bipartite graph $G$ is maximized when we choose a bipartite orientation for $G$. We will find an injective function from the set of linear extensions of any fixed acyclic orientation to the set of linear extensions of a bipartite orientation, in such a way that the defined map is surjective if and only if the initial orientation is bipartite. Throughout the section, let $G$ be bipartite with $n \geq 1$ vertices.

Definition 2.1 Suppose that $G=G(V, E)$ has a bipartition $V=V_{1} \sqcup V_{2}$. Then, the orientations that either choose $\left(v_{1}, v_{2}\right)$ for all $\left\{v_{1}, v_{2}\right\} \in E$ with $v_{1} \in V_{1}$ and $v_{2} \in V_{2}$, or $\left(v_{2}, v_{1}\right)$ for all $\left\{v_{1}, v_{2}\right\} \in E$ with $v_{1} \in V_{1}$ and $v_{2} \in V_{2}$, are called bipartite orientations of $G$.

Definition 2.2 For a graph $G$ on vertex set $V$ with $|V|=n$, we will denote by $B i j(V,[n])$ the set of bijections from $V$ to $[n]$. 
To begin, we will define a special set of automorphisms of $\operatorname{Bij}(V,[n])$ that will serve as building blocks for constructing our function.

Definition 2.3 Consider a simple graph $G=G(V, E)$ with $|V|=n$. For different vertices $u, v \in V$, let rev $_{u v}$ be the automorphism of $\operatorname{Bij}(V,[n])$ given by the following rule: For all $f \in B i j(V,[n])$, let

$$
\begin{array}{ll}
\operatorname{rev}_{u v}(f)(u) & =f(v) \\
\operatorname{rev}_{u v}(f)(v) & =f(u) \\
\operatorname{rev}_{u v}(f)(w) & =f(w) \text { if } w \in V \backslash\{u, v\} .
\end{array}
$$

It is clear that $\left(\operatorname{rev}_{u v} \circ \operatorname{rev}_{u v}\right)(f)=f$ for all $f \in \operatorname{Bij}(V,[n])$. Moreover, we will need the following technical observation about $\operatorname{rev}_{u v}$.

Observation 2.4 Let $G=G(V, E)$ be a simple graph with $|V|=n$ and consider a bijection $f \in$ $\operatorname{Bij}(V,[n])$. Then, if for some $u, v, x, y \in V$ with $f(u)<f(v)$ we have that rev $u v(f)(x)>\operatorname{rev}_{u v}(f)(y)$ but $f(x)<f(y)$, then $f(u) \leq f(x)<f(y) \leq f(v)$ and furthermore, at least one of $f(x)$ or $f(y)$ must be equal to one of $f(u)$ or $f(v)$.

Let us present the main result of this section:

Theorem 2.5 Let $G=G(V, E)$ be a connected bipartite simple graph with $|V|=n$, and with bipartite orientations $O_{\text {down }}$ and $O_{\text {up }}$. Let also $O$ be an acyclic orientation of $G$. Then, there exists an injective function $\Theta$ from the set of linear extensions of $O$ to the set of linear extensions of $O_{u p}$ and furthermore, $\Theta$ is surjective if and only if $O=O_{u p}$ or $O=O_{\text {down. }}$.

Proof: Let $f$ be a linear extension of $O$, and without loss of generality assume that $O \neq O_{\text {up }}$. We seek to find a function $\Theta$ that transforms $f$ into a linear extension of $O_{\text {up }}$ injectively. The idea will be to describe how $\Theta$ acts on $f$ as a composition of automorphisms of the kind presented in Definition 2.3. Now, we will find the terms of the composition in an inductive way, where at each step we consider the underlying configuration obtained after the previous steps. In particular, the choice of terms in the composition will depend on $f$. The inductive steps will be indexed using a positive integer variable $k$, starting from $k=1$, and at each step we will know an acyclic orientation $O_{k}$ of $G$, a set $B_{k}$ and a function $f_{k}$. The set $B_{k} \subseteq V$ will always be defined as the set of all vertices incident to an edge whose orientation in $O_{k}$ and $O_{\text {up }}$ differs, and $f_{k}$ will be a particular linear extension of $O_{k}$ that we will define.

Initially, we set $O_{1}=O$ and $f_{1}=f$, and calculate $B_{1}$. Now, suppose that for some fixed $k \geq 1$ we know $O_{k}, B_{k}$ and $f_{k}$, and we want to compute $O_{k+1}, B_{k+1}$ and $f_{k+1}$. If $B_{k}=\emptyset$, then $O_{k}=O_{\text {up }}$ and $f_{k}$ is a linear extension of $O_{\text {up }}$, so we stop our recursive process. If not, then $B_{k}$ contains elements $u_{k}$ and $v_{k}$ such that $f_{k}\left(u_{k}\right)$ and $f_{k}\left(v_{k}\right)$ are respectively minimal and maximal elements of $f_{k}\left(B_{k}\right) \subseteq[n]$. Moreover, $u_{k} \neq v_{k}$. We will then let $f_{k+1}:=\operatorname{rev}_{u_{k} v_{k}}\left(f_{k}\right), O_{k+1}$ be the acyclic orientation of $G$ induced by $f_{k+1}$, and calculate $B_{k+1}$ from $O_{k+1}$.

If we let $m$ be the minimal positive integer for which $B_{m+1}=\emptyset$, then $\Theta(f)=\left(\operatorname{rev}_{u_{m} v_{m}} \circ \cdots \circ\right.$ $\left.\operatorname{rev}_{u_{2} v_{2}} \circ \operatorname{rev}_{u_{1} v_{1}}\right)(f)$. The existence of $m$ follows from observing that $B_{k+1} \subsetneq B_{k}$ whenever $B_{k} \neq \emptyset$. In particular, if $B_{k} \neq \emptyset$, then $u_{k}, v_{k} \in B_{k} \backslash B_{k+1}$ and so $1 \leq m \leq\left\lfloor\frac{\left|B_{1}\right|}{2}\right\rfloor$. It follows that the pairs $\left\{\left\{u_{k}, v_{k}\right\}\right\}_{k \in[m]}$ are pairwise disjoint, $f\left(u_{k}\right)=f_{k}\left(u_{k}\right)$ and $f\left(v_{k}\right)=f_{k}\left(v_{k}\right)$ for all $k \in[m]$, and $f\left(u_{1}\right)<$ $f\left(u_{2}\right)<\cdots<f\left(u_{m}\right)<f\left(v_{m}\right)<\cdots<f\left(v_{2}\right)<f\left(v_{1}\right)$. As a consequence, the automorphisms in the 
composition description of $\Theta$ commute. Lastly, $f_{m+1}$ will be a linear extension of $O_{\text {up }}$ and we stop the inductive process by defining $\Theta(f)=f_{m+1}$.

To prove that $\Theta$ is injective, note that given $O$ and $f_{m+1}$ as above, we can recover uniquely $f$ by imitating our procedure to find $\Theta(f)$. First, set $g_{1}:=f_{m+1}$ and $Q_{1}:=O_{\text {up }}$, and compute $C_{1} \subseteq V$ as the set of vertices incident to an edge whose orientation differs in $Q_{1}$ and $O$. Assuming prior knowledge of $Q_{k}, C_{k}$ and $g_{k}$, and whenever $C_{k} \neq \emptyset$ for some positive integer $k$, find the elements of $C_{k}$ whose images under $g_{k}$ are maximal and minimal in $g_{k}\left(C_{k}\right)$. By the discussion above and Observation 2.4, we check that these are respectively and precisely $u_{k}$ and $v_{k}$. Resembling the previous case, we will then let $g_{k+1}:=\operatorname{rev}_{u_{k} v_{k}}\left(g_{k}\right), Q_{k+1}$ be the acyclic orienation of $G$ induced by $g_{k}$, and compute $C_{k+1}$ accordingly as the set of vertices incident to an edge with different orientation in $Q_{k+1}$ and $O$. Clearly $g_{m+1}=f$, and the procedure shows that $\Theta$ is invertible in its image.

To establish that $\Theta$ is not surjective whenever $O \neq O_{\text {down }}$, note that then $O$ contains a directed 2-path $(w, u)$ and $(u, v)$. Without loss of generality, we may assume that the orientation of these edges in $O_{\text {up }}$ is given by $(w, u)$ and $(v, u)$. But then, a linear extension $g$ of $O_{\text {up }}$ in which $g(u)=n$ and $g(v)=1$ is not in $\operatorname{Im}(\Theta)$ since otherwise, using the notation and framework discussed above, there would exist different $i, j \in[m]$ such that $u_{i}=u$ and $v_{j}=v$, which then contradicts the choice of $u_{1}$ and $v_{1}$. This completes the proof.

\section{Odd Cycles.}

In this section $G=G(V, E)$ will be a cycle on $2 n+1$ vertices with $n \geq 1$. The case of odd cycles follows as an immediate extension of the case of bipartite graphs, but it will also be covered under a different guise in Section 5 Optimal acyclic orientations of odd cycles resemble as much as possible bipartite orientations:

Definition 3.1 For an odd cycle $G=G(V, E)$, we say that an acyclic orientation of its edges is almost bipartite if under the orientation there exists exactly one directed 2-path, i.e. only one instance of $(u, v)$ and $(v, w)$ with $u, v, w \in V$.

Theorem 3.2 Let $G=G(V, E)$ be an odd cycle on $2 n+1$ vertices with $n \geq 1$. Then, the acyclic orientations of $E$ that maximize the number of linear extensions are the almost bipartite orientations.

Proof (First proof): Since the case when $n=1$ is straightforward let us assume that $n \geq 2$, and consider an arbitrary acyclic orientation $O$ of $G$. Again, our method will be to construct an injective function $\Theta^{\prime}$ that transforms every linear extension of $O$ into a linear extension of some fixed almost bipartite orientation of $G$, where the specific choice of almost bipartite orientation will not matter by the symmetry of $G$.

To begin, note that there must exist a directed 2-path in $O$, say $(u, v)$ and $(v, w)$ for some $u, v, w \in V$. Our goal will be to construct $\Theta^{\prime}$ so that it maps into the set of linear extensions of the almost bipartite orientation $O_{u v w}$ in which our directed path $(u, v),(v, w)$ is the unique directed 2-path. To find $\Theta^{\prime}$, first consider the bipartite graph $G^{\prime}$ with vertex set $V \backslash\{v\}$ and edge set $E \backslash(\{u, v\} \cup\{v, w\}) \cup\{u, w\}$, along with the orientation $O^{\prime}$ of its edges that agrees on common edges with $O$ and contains $(u, w)$. Clearly $O^{\prime}$ is acyclic. If $f$ is a linear extension of $O$, we regard the restriction $f^{\prime}$ of $f$ to $V \backslash\{v\}$ as a strict orderpreserving map on $O^{\prime}$, and analogously to the proof of Theorem 2.5. we can transform injectively $f^{\prime}$ into a strict order-preseving map $g^{\prime}$ with $\operatorname{Im}\left(g^{\prime}\right)=\operatorname{Im}\left(f^{\prime}\right)=\operatorname{Im}(f) \backslash\{f(v)\}$ of the bipartite orientation of 
$G^{\prime}$ that contains $(u, w)$. Now, if we define $g \in \operatorname{Bij}(V,[n])$ via $g(x)=g^{\prime}(x)$ for all $x \in V \backslash\{v\}$ and $g(v)=f(v)$, we see that $g$ is a linear extension of $O_{u v w}$. We let $\Theta^{\prime}(f)=g$.

The technical work for proving the general injectiveness of $\Theta^{\prime}$, and its non-surjectiveness when $O$ is not almost bipartite, has already been presented in the proof of Theorem 2.5. That $\Theta^{\prime}$ is injective follows from the injectiveness of the map transforming $f^{\prime}$ into $g^{\prime}$, and then by noticing that $f(v)=g(v)$. Nonsurjectiveness follows from noting that if $O$ is not almost bipartite, then $O$ contains a directed 2-path $(a, b),(b, c)$ with $a, b, c \in V$ and $b \neq v$, so we cannot have simultaneously $g^{\prime}(a)=\min \operatorname{Im}\left(f^{\prime}\right)$ and $g^{\prime}(c)=\max \operatorname{Im}\left(f^{\prime}\right)$.

\section{Comparability graphs.}

In this section, we will study our main problem using more general tehniques. As a consequence, we will be able to understand the case of comparability graphs, which includes bipartite graphs as a special case: The acyclic orientations of the edges of a comparability graph $G$ that maximize the number of linear extensions are precisely the orientations that induce posets whose comparability graph agrees with $G$. Recall that a comparability graph is a simple undirected graph $G=G(V, E)$ for which there exists a partial order on $V$ under which two different vertices $u, v \in V$ are comparable if and only if $\{u, v\} \in E$.

Comparability graphs have been largely discussed in the literature, mainly due to their connection with partial orders and because they are perfectly orderable graphs and more generally, perfect graphs. Comparability graphs, perfectly orderable graphs and perfect graphs are all large hereditary classes of graphs. In Gallai's fundamental work in Ramírez-Alfonsín and Reed (2001), a characterization of comparability graphs in terms of forbidden subgraphs was given and the concept of modular decomposition of a graph was introduced.

Note that given a comparability graph $G=G(V, E)$, we can find at least two partial orders on $V$ induced by acyclic orientations of $E$ whose comparability graphs (obtained as discussed above) agree precisely with $G$, and the number of such posets depends on the modular structure of $G$. Let us record this idea in a definition.

Definition 4.1 Let $G=G(V, E)$ be a comparability graph, and let $O$ be an acyclic orientation of $E$ such that the comparability graph of the partial order of $V$ induced by $O$ agrees precisely with $G$. Then, we will say that $O$ is a transitive orientation of $G$.

We will present two methods for proving our main result. The first one relies on Stanley's transfer map between the order polytope and the chain polytope of a poset, and the second one is made possible by relating our problem to network flows.

To begin, let us recall the main definitions and notation related to the first method.

Definition 4.2 We will consider $\mathbb{R}^{n}$ with euclidean topology, and let $\left\{e_{j}\right\}_{j \in[n]}$ be the standard basis of $\mathbb{R}^{n}$. For $J \subseteq[n]$, we will define $e_{J}:=\sum_{j \in J} e_{j}$ and $e_{\emptyset}:=0$; furthermore, for $x \in \mathbb{R}^{n}$ we will let $x_{J}:=\sum_{j \in J} x_{j}$ and $x_{\emptyset}:=0$.

Definition 4.3 Given a partial order $P$ on $[n]$, the order polytope of $P$ is defined as:

$$
\mathcal{O}(P):=\left\{x \in \mathbb{R}^{n}: 0 \leq x_{i} \leq 1 \text { and } x_{j} \leq x_{k} \text { whenever } j \leq_{P} k, \forall i, j, k \in[n]\right\} .
$$

The chain polytope of $P$ is defined as:

$$
\mathcal{C}(P):=\left\{x \in \mathbb{R}^{n}: x_{i} \geq 0, \forall i \in[n] \text { and } x_{C} \leq 1 \text { whenever } C \text { is a chain in } P\right\} .
$$


Stanley's transfer map $\phi: \mathcal{O}(P) \rightarrow \mathcal{C}(P)$ is the function given by $\phi(x)_{i}=x_{i}-\max _{j{ }_{i} i} x_{j}$ for all $i \in[n]$ and $x \in \mathcal{O}(P)$.

Let $P$ be a partial order on $[n]$. It is easy to see from the definitions that the vertices of $\mathcal{O}(P)$ are given by all the $e_{I}$ with $I$ an order filter of $P$, and those of $\mathcal{C}(P)$ are given by all the $e_{A}$ with $A$ an antichain of $P$.

Now, a well-known result of Stanley $(1986)$ is that $\operatorname{Vol}(\mathcal{O}(P))=\frac{1}{n !} e(P)$ where $e(P)$ is the number of linear extensions of $P$. This result can be proved by considering the unimodular triangulation of $\mathcal{O}(P)$ whose maximal (closed) simplices have the form $\Delta_{\sigma}:=\left\{x \in \mathbb{R}^{n}: 0 \leq x_{\sigma^{-1}(1)} \leq x_{\sigma^{-1}(2)} \leq \cdots \leq\right.$ $\left.x_{\sigma^{-1}(n)} \leq 1\right\}$ with $\sigma: P \rightarrow \mathbf{n}$ a linear extension of $P$. However, the volume of $\mathcal{C}(P)$ is not so direct to compute. To find $\operatorname{Vol}(\mathcal{C}(P))$ Stanley made use of the transfer map $\phi$, a pivotal idea that we now wish to describe in detail since it will provide a geometrical point of view on our main problem.

It is easy to see that $\phi$ is invertible and its inverse can be described by:

$$
\phi^{-1}(x)_{i}=\max _{\substack{C \text { chain in } P: \\ i \text { is maximal in } C}} x_{C} \text {, for all } i \in[n] \text { and } x \in \mathcal{C}(P) .
$$

As a consequence, we see that $\phi^{-1}\left(e_{A}\right)=e_{A} \vee$ for all antichains $A$ of $P$, where $A^{\vee}$ is the order filter of $P$ induced by $A$. It is also straightforward to notice that $\phi$ is linear on each of the $\Delta_{\sigma}$ with $\sigma$ a linear extension of $P$, by staring at the definition of $\Delta_{\sigma}$. Hence, for fixed $\sigma$ and each $i \in[n]$, we can consider the order filters $A_{i}^{\vee}:=\sigma^{-1}([i, n])$ along with their respective minimal elements $A_{i}$ in $P$, and notice that $\phi\left(e_{A_{i}^{\vee}}\right)=e_{A_{i}}$, and also that $\phi(0)=0$. From there, $\phi$ is now easily seen to be a unimodular linear map on $\Delta_{\sigma}$, and so $\operatorname{Vol}\left(\phi\left(\Delta_{\sigma}\right)\right)=\operatorname{Vol}\left(\Delta_{\sigma}\right)=\frac{1}{n !}$. Since $\phi$ is invertible, without unreasonable effort we have obtained the following central result:

Theorem 4.4 (Stanley (1986)) Let $P$ be a partial order on $[n]$. Then, $\operatorname{Vol}(\mathcal{O}(P))=\operatorname{Vol}(\mathcal{C}(P))=$ $\frac{1}{n !} e(P)$, where $e(P)$ is the number of linear extensions of $P$.

Now, recall that the stable polytope $\operatorname{stab}(G)$ of a simple undirected graph $G=G(V, E)$ is the convex hull in $\left(\mathbb{R}^{V}\right)^{*}$ of the indicator functions of the stable sets of $G$. Therefore, the chain polytope of a partial order $P$ on $[n]$ is equal to $\operatorname{stab}(G)$ of its comparability graph $G=G([n], E)$ since antichains of $P$ correspond to stable sets of $G$. In combination with Theorem 4.4 this shows that the number of linear extensions is a comparability invariant, i.e. two posets with isomorphic comparability graphs have the same number of linear extensions.

We are now ready to present the first proof of the main result for comparability graphs.

Theorem 4.5 Let $G=G(V, E)$ be a connected comparability graph. Then, the acyclic orientations of $E$ that maximize the number of linear extensions are exactly the transitive orientations of $G$.

Proof (First proof): Without loss of generality, assume that $V=[n]$. Let $O$ be an acyclic orientation of $G$ inducing a partial order $P$ on $[n]$. If two vertices $i, j \in[n]$ are incomparable in $P$, then $\{i, j\} \notin E$. This implies that all antichains of $P$ are stable sets of $G$, and so $\mathcal{C}(P) \subseteq \operatorname{stab}(G)$.

On the other hand, if $O$ is not transitive, then there exists two vertices $k, \ell \in[n]$ such that $\{k, \ell\} \notin E$, but such that $k$ and $\ell$ are comparable in $P$, i.e. the transitive closure of $O$ induces comparability of $k$ and $\ell$. Then, $e_{k}+e_{\ell}$ is a vertex of the stable polytope $\operatorname{stab}(G)$ of $G$, but since $\mathcal{C}(P)$ is a subpolytope of the $n$-dimensional cube, $e_{k}+e_{\ell} \notin \mathcal{C}(P)$. We obtain that $\mathcal{C}(P) \neq \operatorname{stab}(G)$ if $O$ is not transitive, and so $\mathcal{C}(P) \subsetneq \operatorname{stab}(G)$. 
If $O$ is transitive, then $\mathcal{C}(P)=\operatorname{stab}(G)$. This completes the proof.

Let us now introduce the background necessary to present our second method. This will eventually lead to a different proof of Theorem 4.5

Definition 4.6 If we consider a simple connected undirected graph $G=G(V, E)$ and endow it with an acyclic orientation of its edges $O$, we will say that $G$ is an oriented graph and consider it (by a slight abuse of notation) a directed graph, so that every member of $E$ is regarded as an ordered pair.

Definition 4.7 Let $G=G(V, E)$ be an oriented graph. We will denote by $\hat{G}$ the oriented graph with vertex set $\hat{V}:=V \cup\{\hat{0}, \hat{1}\}$ and set of directed edges $\hat{E}$ equal to the union of $E$ and all edges of the form:

$$
\begin{aligned}
& (v, \hat{1}) \text { with } v \in V \text { and outdeg }(v)=0 \text { in } G, \text { and } \\
& (\hat{0}, v) \text { with } v \in V \text { and indeg }(v)=0 \text { in } G .
\end{aligned}
$$

A natural flow on $G$ will be a function $f: \hat{E} \rightarrow \mathbb{R}_{\geq 0}$ such that for all $v \in V$, we have:

$$
\sum_{(x, v) \in \hat{E}} f(x, v)=\sum_{(v, y) \in \hat{E}} f(v, y)
$$

In other words, a natural flow on $G$ is a nonnegative network flow on $\hat{G}$ with unique source $\hat{0}$ and unique sink $\hat{1}$, and with infinite capacities in all edges.

First, let us relate natural flows on oriented graphs with linear extensions of induced posets.

Lemma 4.8 Let $G=G(V, E)$ be an oriented graph with induced partial order $P$ on $V$, with $|V|=n$. Then, the function $g: \hat{E} \rightarrow \mathbb{R}_{\geq 0}$ defined by

$$
\begin{aligned}
g(u, v) & =\mid\{\sigma: \sigma \text { is a linear extension of } P \text { and } \sigma(u)=\sigma(v)-1\} \mid \\
& \text { if }(u, v) \in E, \\
g(v, \hat{1}) \quad=\mid\{\sigma: \sigma \text { is a linear extension of } P \text { and } \sigma(v)=n\} \mid & \text { if } v \in V \text { and outdeg }(v)=0 \text { in } G, \text { and } \\
g(\hat{0}, v) \quad=\mid\{\sigma: \sigma \text { is a linear extension of } P \text { and } \sigma(v)=1\} \mid & \text { if } v \in V \text { and indeg }(v)=0 \text { in } G,
\end{aligned}
$$

is a natural flow on G. Moreover, the net $g$-flow from $\hat{0}$ to $\hat{1}$ is equal to e $(P)$.

Proof: Assume without loss of generality that $V=[n]$, and consider the directed graph $K$ on vertex set $V(K)=[n] \cup\{\hat{0}, \hat{1}\}$ whose set $E(K)$ of directed edges consists of all:

$$
\begin{aligned}
(i, j) & \text { for } i<_{P} j, \\
(i, j) \text { and }(j, i) & \text { for } i \|_{P} j, \\
(\hat{0}, i) & \text { for } i \text { minimal in } P, \text { and } \\
(i, \hat{1}) & \text { for } i \text { maximal in } P .
\end{aligned}
$$

As directed graphs, we check that $\hat{G}$ is a subgraph of $K$. We will define a network flow on $K$ with unique source $\hat{0}$ and unique sink $\hat{1}$, expressing it as a sum of simpler network flows. 
First, extend each linear extension $\sigma$ of $P$ to $V(K)$ by further defining $\sigma(\hat{0})=0$ and $\sigma(\hat{1})=n+1$. Then, let $f_{\sigma}: E(K) \rightarrow \mathbb{R}_{\geq 0}$ be given by

$$
f_{\sigma}(x, y)= \begin{cases}1 & \text { if } \sigma(x)=\sigma(y)-1 \\ 0 & \text { otherwise }\end{cases}
$$

Clearly, $f_{\sigma}$ defines a network flow on $K$ with source $\hat{0}$, sink $\hat{1}$, and total net flow 1 , and then $f:=$ $\sum_{\sigma \text { linear ext. of } P} f_{\sigma}$ defines a network flow on $K$ with total net flow $e(P)$. Moreover, for each $(x, y) \in \hat{E}$ we have that $f(x, y)=g(x, y)$. It remains now to check that the restriction of $f$ to $\hat{E}$ is still a network flow on $\hat{G}$ with total flow $e(P)$.

We have to verify two conditions. First, for $i, j \in[n]$ and if $i \|_{P} j$, then

$$
\begin{aligned}
& \mid\{\sigma: \sigma \text { is a lin. ext. of } P \text { and } \sigma(i)=\sigma(j)-1\} \mid \\
& =\mid\{\sigma: \sigma \text { is a lin. ext. of } P \text { and } \sigma(j)=\sigma(i)-1\} \mid \text {, }
\end{aligned}
$$

so $f(i, j)=f(j, i)$, i.e. the net $f$-flow between $i$ and $j$ is 0 . Second, again for $i, j \in[n]$, if $i<_{P} j$ but $i \nless{ }_{P} j$, then $f(i, j)=0$. These two observations imply that $g$ defines a network flow on $\hat{G}$ with total flow $e(P)$.

Corollary 4.9 Let $P$ be a partial order on $V$, with $|V|=n$. If $A$ is an antichain of $P$, then $e(P) \geq$ $\sum_{v \in A} e(P \backslash v)$, where $P \backslash v$ denotes the induced poset on $V \backslash\{v\}$. Similarly, if $S$ is a cutset of $P$, then $e(P) \leq \sum_{v \in S} e(P \backslash v)$. Moreover, if $I$ is a subset $V$ that is either a cutset or an antichain of $P$, then $e(P)=\sum_{v \in I} e(P \backslash v)$ if and only if $I$ is both a cutset and an antichain of $P$.

Proof: Let $G=G(V, E)$ be any graph that contains as a subgraph the Hasse diagram of $P$, and oriented so that it induces exactly $P$. Let $g$ be as in Lemma 4.8 Since edges representing cover relations of $P$ are in $G$, the net $g$-flow is $e(P)$. Moreover, by the standard chain decomposition of network flows of Ford Jr and Fulkerson (2010) (essentially Stanley's transfer map), which expresses $g$ as a sum of positive flows through each maximal chain of $P$, it is clear that for $A$ an antichain of $P$, we have that $e(P) \geq \sum_{v \in A} \sum_{(x, v) \in \hat{E}} g(x, v)$, since antichains intersect maximal chains of $P$ at most once. Similarly, for $S$ a cutset of $P$, we have that $e(P) \leq \sum_{v \in S} \sum_{(x, v) \in \hat{E}} g(x, v)$ since every maximal chain of $P$ intersects $S$. Furthermore, equality will only hold in either case if the other case holds as well. But then, for each $v \in V$, the map Trans that transforms linear extensions of $P \backslash v$ into linear extensions of $P$ and defined via: For $\sigma$ a linear extension of $P \backslash v$ and $\kappa:=\max _{y<P v} \sigma(y)$,

$$
\operatorname{Trans}(\sigma)(x)= \begin{cases}\kappa+1 & \text { if } x=v \\ \sigma(x)+1 & \text { if } \sigma(x)>\kappa \\ \sigma(x) & \text { otherwise }\end{cases}
$$

is a bijection onto its image, and the number $\sum_{(x, v) \in \hat{E}} g(x, v)$ is precisely $|\operatorname{Im}(\operatorname{Trans})|$.

Getting ready for the second proof of Theorem 4.5 , it will be useful to have a notation for the main object of study in this paper: 
Definition 4.10 Let $G=G(V, E)$ be an undirected simple graph. The maximal number of linear extensions of a partial order on $V$ induced by an acyclic orientation of $E$ will be denoted by $\varepsilon(G)$.

Proof (Second proof of Theorem 4.5): Assume without loss of generality that $V=[n]$. We will do induction on $n$. The case $n=1$ is immediate, so assume the result holds for $n-1$. Note that every induced subgraph of $G$ is also a comparability graph and moreover, every transitive orientation of $G$ induces a transitive orientation on the edges of every induced graph of $G$. Now, let $O$ be a non-transitive orientation of $E$ with induced poset $P$, so there exists a comparable pair $\{k, \ell\}$ in $P$ that is stable in $G$. Let $S$ be an antichain cutset of $P$. Then, $S$ is a stable set of $G$, and letting $G \backslash i$ be the induced subgraph of $G$ on vertex set $[n] \backslash\{i\}$, we obtain that $\varepsilon(G) \geq \sum_{i \in S} \varepsilon(G \backslash i) \geq \sum_{i \in S} e(P \backslash i)=e(P)$, where the first inequality is an application of Corollary 4.9 on a transitive orientation of $G$, along with Definition 4.10 and the inductive hypothesis, the second inequality is obtained after recognizing that the poset induced by $O$ on each $G \backslash i$ is a subposet of $P \backslash i$ and by Definition 4.10, and the last equality follows because $S$ is a cutset of $P$. If $|S|>1$ or $S \cap\{k, \ell\}=\emptyset$, then by induction the second inequality will be strict. On the other hand, if $S=\{k\}$ or $S=\{\ell\}$, then the first inequality will be strict since $\{k, \ell\}$ is stable in $G$.

Lastly, the different posets arising from transitive orientations of $G$ have in common that their antichains are exactly the stable sets of $G$, and their cutsets are exactly the sets that meet every maximal clique of $G$ at least once, so by the corollary, the inductive hypothesis and our choice of $S$ before, these posets have the same number of linear extensions and this number is in general at least $\sum_{i \in S} \varepsilon(G \backslash i)$, and strictly greater if $S=\{k\}$ or $S=\{\ell\}$.

\section{Beyond comparability.}

The goal of this section is to illustrate applications of the ideas developed in Section 4 First, we will obtain once more the result for odd cycles. Then, we will use an approximation technique for the enumeration problem in comparability graphs and general graphs.

From Theorem 4.5 we can say the following:

Observation 5.1 Let $P$ and $Q$ be partial orders on the same ground set, and suppose that the comparability graph of $P$ contains as a subgraph the comparability graph of $Q$. Then, $e(Q) \geq e(P)$ and moreover, if the containment of graphs is proper, then $e(Q)>e(P)$.

Proof (Second proof of Theorem 3.2): Note that every acyclic orientation $O$ of $E$ induces a partial order on $V$ whose comparability graph contains (as a subgraph) the comparability graph of a poset given by an almost bipartite orientation, and this containment is proper if $O$ is not almost bipartite. By the symmetry of $G$, then all of the almost bipartite orientations are equivalent.

Note to proof: The same technique allows us to obtain results for other restrictive families of graphs, like odd cycles with isomorphic trees similarly attached to every element of the cycle, but we do not pursue this here.

Let us now turn our attention to the enumeration problem. We will first include here a result for comparability graphs, which will serve as a conceptual preamble for the remaining part of this section.

Theorem 5.2 Let $G=G(V, E)$ be a comparability graph, and further let $V=\left\{v_{1}, v_{2}, \ldots, v_{n}\right\}$. For $u_{1}, u_{2}, \ldots, u_{k} \in V$, let $G \backslash u_{1} u_{2} \ldots u_{k}$ be the induced subgraph of $G$ on vertex set $V \backslash\left\{u_{1}, u_{2}, \ldots, u_{k}\right\}$. 
Then,

$$
\varepsilon(G) \geq \sum_{\sigma \in \mathfrak{S}_{n}} \frac{1}{\chi(G) \chi\left(G \backslash v_{\sigma 1}\right) \chi\left(G \backslash v_{\sigma 1} v_{\sigma 2}\right) \chi\left(G \backslash v_{\sigma 1} v_{\sigma 2} v_{\sigma 3}\right) \ldots \chi\left(v_{\sigma n}\right)},
$$

where $\mathfrak{S}_{n}$ denotes the symmetric group on $[n]$ and $\chi$ denotes the chromatic number of the graph.

Proof: Let us first fix a perfect order $\omega$ of the vertices of $G$, i.g. $\omega$ can be a linear extension of a partial order on $V$ whose comparability graph is $G$. Let $H$ be an induced subgraph of $G$ with vertex set $V(H)$ and edge set $E(H)$, let $\omega_{H}$ be the restriction of $\omega$ to $V(H)$, and let $Q$ be the partial order of $V(H)$ given by labeling every $v \in V(H)$ with $\omega_{H}(v)$ and orienting $E(H)$ accordingly. Using the colors of the optimal coloring of $H$ given by $\omega_{H}$, we can find $\chi(H)$ mutually disjoint antichains of $Q$ that cover $Q$, so by Corollary 4.9 we obtain that

$$
e(Q) \geq \frac{1}{\chi(H)} \sum_{v \in V(H)} e(Q \backslash v) .
$$

Now, we note that each $Q \backslash v$ with $v \in V(H)$ is also induced by the respective restriction of $\omega$ to $V(H) \backslash v$, and that the comparability of $Q \backslash v$ is $H \backslash v$, and then each of the terms on the right hand side can be expanded similarly. Starting from $H=G$ above and noting the fact that $\varepsilon(G)=e(Q)$ for this case, we can expand the terms of 5.1 exhaustively to obtain the desired expression.

Corollary 5.3 Let $G=G(V, E)$ be any graph on $n$ vertices with chromatic number $k:=\chi(G)$. Then $\varepsilon(G) \geq \frac{n !}{k^{n-k} k !}$.

Proof: We can follow the proof of Theorem 5.2 This time, starting from $H=G, Q$ will be a poset on $V$ given by a minimal coloring of $G$, i.e. we color $G$ using a minimal number of totally ordered colors and orient $E$ accordingly. Then, $\varepsilon(G) \geq e(Q)$ and we can expand the right hand side of 5.1, but noting that $Q \backslash v$ can only be guaranteed to be partitioned into at most $\chi(G)$ antichains, and that the chromatic number of a graph is at most the number of vertices of that graph.

Noting that the number of cutsets is a least 2 in most cases, a similar argument to that of Theorem 5.2 implies:

Observation 5.4 Let $G=G(V, E)$ be a connected graph. Then:

$$
\varepsilon(G) \leq \frac{1}{2} \sum_{v \in V} \varepsilon(G \backslash v) .
$$

Example 1 If $G=G(V, E)$ is the odd cycle on $2 n+1$ vertices, then for each $v \in V$ we have $\varepsilon(G \backslash v)=$ $E_{2 n}$, the $(2 n)$-th Euler number, and $\chi(G)=3$, so $a_{n}:=\frac{(2 n+1) E_{2 n}}{2} \geq \varepsilon(G) \geq b_{n}:=\frac{(2 n+1) !}{3^{2 n-2} \cdot 3 !}$. As $n$ goes to infinity, then $\frac{a_{n}}{b_{n}} \sim \frac{4}{3 \pi}\left(\frac{6}{\pi}\right)^{2 n}$. 


\section{Further techniques.}

In this section, we will see how our problem has two more presentations as selecting a region in the graphical arrangement with maximal fractional volume, or as selecting a vertex of the graphical zonotope which is farthest from the origin in euclidean distance.

Definition 6.1 Consider a simple undirected graph $G=G([n], E)$. The graphical arrangement of $G$ is the central hyperplane arrangement in $\mathbb{R}^{n}$ given by:

$$
\mathcal{A}_{G}=\left\{x \in \mathbb{R}^{n}: x_{i}-x_{j}=0, \forall\{i, j\} \in E\right\} .
$$

The regions of the graphical arrangement $\mathcal{A}_{G}$ with $G=G([n], E)$ are in one-to-one correspondence with the acyclic orientations of $G$. Moreover, the complete fan in $\mathbb{R}^{n}$ given by $\mathcal{A}_{G}$ is combinatorially dual to the graphical zonotope of $G$ :

$$
\mathcal{Z}(G):=\sum_{\{i, j\} \in E}\left[e_{i}-e_{j}, e_{j}-e_{i}\right],
$$

and there is a clear correspondence between regions of $\mathcal{A}_{G}$ and vertices of $\mathcal{Z}(G)$.

Following Klivans and Swartz (2011), we define the fractional volume of a region $\mathcal{R}$ of $\mathcal{A}_{G}$ to be: $\operatorname{Vol}^{\circ}(\mathcal{R})=\frac{\operatorname{Vol}\left(B^{n} \cap \mathcal{R}\right)}{\operatorname{Vol}\left(B^{n}\right)}$, where $B^{n}$ is the unit $n$-dimensional ball in $\mathbb{R}^{n}$.

With little work it is possible to say the following about these volumes:

Proposition 6.2 Let $G=G([n], E)$ be an undirected simple graph, and let $\mathcal{A}_{G}$ be its graphical arrangement. If $\mathcal{R}$ is a region of $\mathcal{A}_{G}$ and $P$ is its corresponding partial order on $[n]$, then:

$$
\operatorname{Vol}^{\circ}(\mathcal{R})=\frac{e(P)}{n !} .
$$

Lemma 6.3 Let $G=G(V, E)$ be an oriented graph. Then,

$$
\frac{1}{2} \sum_{v \in V}(\operatorname{outdeg}(v)-\operatorname{indeg}(v))^{2}=|E|+\operatorname{tri}(G)+\operatorname{incom}(G)-\operatorname{com}(G),
$$

where tri $(G)$ is the number of directed triangles $(u, v),(v, w),(u, w) \in E$, incom $(G)$ is the number of triples $u, v, w \in V$ such that $(v, w),(w, v) \notin E$ but either $(u, v),(u, w) \in E$ or $(v, u),(w, u) \in E$, and $\operatorname{com}(G)$ is the number of directed 2-paths $(u, v),(v, w) \in E$ such that $(u, w) \notin E$.

Proof: For $v \in V$, outdeg $(v)^{2}$ is equal to outdeg $(v)$ plus two times the number of pairs $u \neq w$ such that $(v, u),(v, w) \in E$, indeg $(v)^{2}$ is equal to indeg $(v)$ plus two times the number of pairs $u, \neq w$ such that $(u, v),(w, v) \in E$, and outdeg $(v) \cdot \operatorname{indeg}(v)$ is equal to the number of pairs $u \neq w$ such that $(u, v),(v, w) \in E$. If we add up these terms and cancel out terms in the case of directed triangles, we obtain the desired equality.

Interestingly enough, for any simple undirected graph $G=G(V, E)$, all the acyclic orientations of $E$ will not vary in their values of $\operatorname{tri}(\cdot)$ and of $|E|$, which depend on $G$, but only in $\operatorname{com}(\cdot)$ and incom $(\cdot)$. Moreover, by Theorem 4.5. for $G$ a comparability graph, the value of incom $(\cdot)-\operatorname{com}(\cdot)$ will be maximized precisely on the transitive orientations of $G$, so by Lemma 6.3 
Corollary 6.4 Let $G=G(V, E)$ be a comparability graph. Then, the vertices of the graphical zonotope of $\mathcal{Z}(G)$ that have maximal euclidean distance to the origin are precisely those that correspond to the transitive orientations of $E$, which in turn have maximal number $\varepsilon(G)$ of linear extensions.

\section{Acknowledgements}

The author would like to specially thank Tanya Khovanova and the Research Science Institute of MIT, Richard Stanley and Carly Klivans, for their strong support and for many helpful discussions.

\section{References}

G. Brightwell and P. Winkler. Counting linear extensions. Order, 8(3):225-242, 1991.

P. Edelman, T. Hibi, and R. P. Stanley. A recurrence for linear extensions. Order, 6(1):15-18, 1989.

L. R. Ford Jr and D. R. Fulkerson. Flows in networks. Princeton Landmarks in Mathematics and Physics. Princeton university press, 2010.

C. J. Klivans and E. Swartz. Projection volumes of hyperplane arrangements. Discrete \& Computational Geometry, 46(3):417-426, 2011.

M. E. Newman. The structure and function of complex networks. SIAM review, 45(2):167-256, 2003.

J. L. Ramírez-Alfonsín and B. A. Reed. Perfect graphs. Series in Discrete Mathematics and Optimization. Wiley-Interscience, 2001.

K. Saito. Principal $\gamma$-cone for a tree. Advances in Mathematics, 212(2):645-668, 2007.

G. Stachowiak. The number of linear extensions of bipartite graphs. Order, 5(3):257-259, 1988.

R. P. Stanley. Two poset polytopes. Discrete \& Computational Geometry, 1(1):9-23, 1986.

R. P. Stanley. Enumerative Combinatorics, Vol. 2:. Cambridge Studies in Advanced Mathematics. Cambridge University Press, 2001.

R. P. Stanley. Enumerative Combinatorics, Vol. 1: Cambridge Studies in Advanced Mathematics. Cambridge University Press, 2011. 